\section{Differenzialdiagnosen der 80 häufigsten Symptome}

— Spüren Sie die klinischen Symptome zielsicher auf und sichern Sie Ihre Diagnose ab: „Pädiatrische Differenzialdiagnostik" bietet praktische Hilfe zur Abklärung von fast 80 häufigen Krankheitssymptomen im Kindes- und Jugendalter.

Nach Funktionssystemen gegliedert, ist jeder Symptomkomplex auf wenigen Seiten übersichtlich und prägnant dargestellt. Das einheitliche Grundgerüst sorgt für eine leichte Orientierung im Buch: Definition, Anamnese, Ätiologie und differenzialdiagnostische Abklärung - Schritt für Schritt und unter Berücksichtigung der Altersstufen. Über 150 Flussdiagramme stellen das
Ursachenspektrum dar und bringen das systematische Vorgehen eingängig auf den Punkt. Die Entscheidungsbäume sind auf einen Blick erfassbar und liefern schnell abrufbare Informationen. Dieses Buch ist ab Anfang 2013 erhältlich.

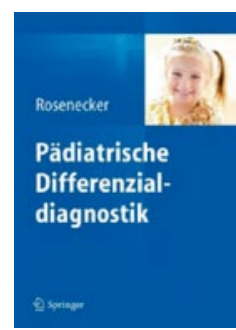

Josef Rosenecker (Hrsg.)

Pädiatrische

Differenzialdiagnostik

Springer, Heidelberg 2013

\title{
Umfassendes Bild der Kinderheilkunde
}

— Sie wollen in der Pädiatrie keine Frage offen lassen? Sie legen Wert auf eine leicht verständliche Sprache, viele Abbildungen und eine übersichtliche Gliederung? Dann machen Sie den „Koletzko" zu Ihrem Lieblingsbuch (Anfang 2013 erhältlich)! Und er bietet Ihnen noch mehr:

_ kurze Einführungen für den ersten Überblick

_ zahlreiche Fallbeispiele in den Kapiteln

_ viele Merksätze für das Allerwichtigste

_ kurze Zusammenfassungen in Lerntabellen und Übersichten
_ ein Fallquiz für noch mehr Praxisnähe

_ eine Übersicht über die Meilensteine der kindlichen Entwicklung

_zuverlässige Normwerttabellen

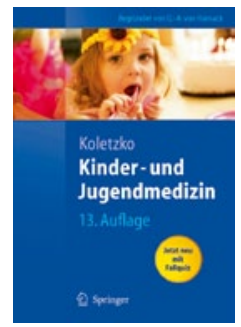

Berthold Koletzko (Hrsg.)

Kinder- und

Jugendmedizin

14. überarb. Aufl.

Springer, Heidelberg

2013

\section{GPGE-Forschungspreis}

Die Gesellschaft für Pädiatrische Gastroenterologie und Ernährung e.V. (GPGE) schreibt für das Jahr 2013 den Paul-Caspar-Tyrell-Forschungspreis aus. Der Verein leberkrankes Kind e. V. stiftet den mit 1.000 Euro dotierten Preis. Ausgezeichnet wird eine wissenschaftliche Arbeit auf dem Gebiet der pädiatrischen Hepatologie. Die Arbeit sollte von besonderem Nutzen für die Behandlung leberkranker und/oder lebertransplantierter Kinder sein.

Die Arbeit muss im Jahre 2011 oder 2012 publiziert oder zur Publikation angenommen worden sein. Die Bewerber sollten in einer Kinderklinik im deutschsprachigen Raum tätig oder von dort beurlaubt sein. Sie sollten vor der Vollendung des 40. Lebensjahres sein. Frauen und Männer, die Elternzeit genommen haben, dürfen pro Kind um zwei Jahre über der Altersgrenze liegen.

Bewerbungen mit Lebenslauf und der Arbeit in sechsfacher Ausfertigung sind bis 15. Januar 2013 zu richten an die

Geschäftsstelle der Gesellschaft für Pädiatrische Gastroenterologie und Ernährung e.V.

Chausseestraße 128-129

10115 Berlin

Der Preis wird anlässlich der 28. Jahrestagung der GPGE (21.-23. März 2013) in Heidelberg und Mannheim überreicht.

\section{Kinderophthalmologie im Blick}

— Kinder müssen das Sehen erst lernen, sie äußern Sehprobleme - wenn überhaupt völlig anders und leiden an ganz eigenen Erkrankungen. Daher benötigen sie bei der Untersuchung und Behandlung auch besondere Aufmerksamkeit. Das vorliegende Werk gibt einen umfassenden und dennoch praxisnahen Überblick über die altersgerechten Untersuchungen, Differenzialdiagnostik und Therapie von Augenerkrankungen im Kindesalter, wie z.B. Amblyopie und Schielen.

Nicht nur Assistenten in der Weiterbildung, Augenärzte, die ihr Wissen vertiefen möchten und Orthoptistinnen, sondern auch Kinderärzte, die sich zur Problematik der
Augenerkrankungen ihrer Patienten fortbilden möchten, finden hier aktuelle Informationen, die mit Tipps zum altersgerechten Vorgehen und Videos ergänzt werden. Dieses Buch ist Anfang nächsten Jahres erhältlich.

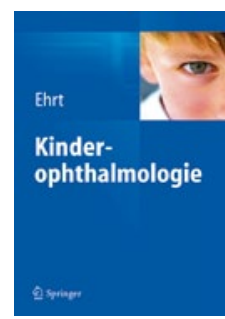

Oliver Ehrt Kinderophthalmologie Springer, Heidelberg 2013 